\title{
Alternative Formulas for Osculatory and Hyperosculatory Inverse Interpolation
}

\author{
By Herbert E. Salzer
}

1. Introduction. In previous papers devoted to inverse osculatory interpolation, all of which were based upon the employment of the Lagrange-Hermite formulas for direct interpolation of $f(x)$ (at equal intervals $h$ for real functions or at points of a Cartesian grid of length $h$ for complex functions) including both the osculatory (function with first derivative) and hyperosculatory (function with first and second derivative) types, the writer has given the Taylor series obtained by the inversion of the direct interpolation series about a suitable point $x_{0}$, in powers of $r=\left[f(x)-f\left(x_{0}\right)\right] / h f^{\prime}\left(x_{0}\right)$ through the term in $r^{10}[1]-[3] .^{*}$ In fact, the same procedure has been employed much earlier for inverse interpolation formulas based upon the ordinary non-osculatory Lagrange interpolation formula, which gives the inversion series in powers of a variable $r$ that is proportional to $f(x)-f\left(x_{0}\right)$ [4]-[6].*

In this present note we give an alternative scheme for inverse osculatory or hyperosculatory interpolation in terms of the inverse function $x(f)$, involving $x(f)$ and $d x(f) / d f$, or $x(f), d x(f) / d f$ and $d^{2} x(f) / d f^{2}$ at separate points $f_{i} \equiv f\left(x_{i}\right)$. In other words, now we distinguish between the previously given point expansions in [1]-[3], which may be characterized as special types of "inverse osculatory or hyperosculatory" formulas and the present "osculatory or hyperosculatory inverse" formulas, which are analogous in structure to the osculatory or hyperosculatory direct interpolation formulas.

\section{Advantages of Alternative Scheme.}

A. The present scheme avoids any cumbersome explicit formulas by applying both a decomposability and uniqueness property of the Lagrange-Hermite interpolation formula, which has been so effective in reducing the labor in direct interpolation of high degree (described in detail in [3] and [7]), to those same kinds of osculatory formulas for the inverse functions.

B. The formulas here, in terms of $x(f)$ with either $x^{\prime}(f)$ or $x^{\prime}(f)$ and $x^{\prime \prime}(f)$ at $f=f_{i}$, enable the user to go far beyond the 10th degree in accuracy with a fraction of the computational labor required for the power series formulas. Actual count of the number of operations for 10th degree accuracy by the older method and 11th degree accuracy (either 6-point osculatory or 4-point hyperosculatory) using the present scheme, showed the latter to involve only around one-fourth of the number of operations required in the former.

C. These alternative formulas are more truly interpolatory because of the actual agreement with the inverse function and its derivatives at different points over the complete range, and the consequent closeness of the approximation near a

Received November 20, 1959; in revised form, March 9, 1960.

* This article is intended to be self-sufficient in the presentation of these alternative schemes for inverse interpolation. We shall avoid as much as possible the repetition of material in [1]-[8] to which the reader is referred for full details. 
number of points; whereas the convergence of the Taylor series expansion becomes rapidly poorer as $|r|$ exceeds 1 .* $^{*}$

D. In this alternative scheme, since the $f_{i}$ 's are the fixed arguments and are unequally spaced, it hardly matters whether the corresponding $x_{i}$ 's are equally or unequally spaced. But for real variables, the previously given formulas in [1] and [3] would not be applicable when the $x_{i}$ 's are irregularly spaced.

E. For complex osculatory or hyperosculatory inverse interpolation for $z=z(f)$ when $f(z)$ is an analytic function tabulated in the complex plane with $f^{\prime}(z)$, or with $f^{\prime}(z)$ and $f^{\prime \prime}(z)$, there is no change in these present formulas other than the replacement of $x$ by $z$.

3. Osculatory and Hyperosculatory Inverse Interpolation Formulas. Before giving the alternative scheme for the osculatory and hyperosculatory cases, we should mention for the sake of completeness that even ordinary inverse interpolation has a corresponding alternative form. Instead of the formulas in [4]-[6], we may prefer the following concise rearrangement of Lagrange's interpolation polynomial for the inverse function $x(f)$ :

$$
x=\sum \alpha_{i} x_{i} / \sum \alpha_{i}
$$

where

$$
\alpha_{i} \equiv A_{i} /\left(f-f_{i}\right)
$$

and

$$
A_{i} \equiv 1 / \prod_{j \neq i}\left(f_{i}-f_{j}\right) .
$$

In the above $(1),\left(2^{\prime}\right),\left(2^{\prime \prime}\right)$, as well as in all subsequent formulas, $x$ denotes either real or complex values, and in any $\sum$ or $\Pi$ the running index $i, j$ or $k$ has whatever range is customary, say $-[(n-1) / 2]$ to $[n / 2]$ for real interpolation or over any set of fixed grid points in complex interpolation (except for omissions indicated beneath the symbol).

For all osculatory and hyperosculatory formulas we employ the first and second derivatives of the inverse function $x(f)$, namely $x^{\prime}(f)=1 / f^{\prime}(x)$ and $x^{\prime \prime}(f)=$ $-f^{\prime \prime}(x) /\left[f^{\prime}(x)\right]^{3}$, at $x=x_{i}$, the same as at $f(x)=f\left(x_{i}\right)$, or more concisely, at $f=f_{i}$. The notation $x_{i}^{\prime}$ and $x_{i}^{\prime \prime}$ is used for $x^{\prime}\left(f_{i}\right)$ and $x^{\prime \prime}\left(f_{i}\right)$ respectively. It is not necessary to repeat here for the inverse function the development given in [3], [7] and [8] for concise expressions for the direct osculatory and hyperosculatory interpolation formulas, since those same ideas apply here.

For the inverse function, in the osculatory and hyperosculatory formulas we

* To see why, say in the real case, in a region where $x(f)$ is one-valued and has derivatives of high enough order, consider the remainder term in the $n$-point osculatory or hyperosculatory Lagrange-Hermite formula for $x(f)$, namely

$$
\left.(1 /(2 n) !)\left\{\Pi_{i-\left[{ }^{(n-1) / 2}\right]}^{[n]}\left(f-f_{i}\right)\right\}^{2}\left(d^{2 n} x /(d f)^{2 n}\right)\right|_{f-f \xi}
$$

or

$$
(1 /(3 n) !)\left\{\prod_{i=-[(n-1) / 2]}^{[n / 2]}\left(f-f_{i}\right)\right\}^{3}\left(d^{3 n} x /(d f)^{3 n}\right) \mid f-f_{\xi}
$$

which can become very small for an $f$ very close to $f_{i}$, even though far from $f_{0}$. 
need the first and second derivatives of $L_{i}{ }^{(n)}(f)$, the $n$-point Lagrange interpolation coefficients in $f$ defined by

$$
L_{i}^{(n)}(f) \equiv \prod_{j \neq i}\left(f-f_{j}\right) / \prod_{j \neq i}\left(f_{i}-f_{j}\right) .
$$

Differentiation is with respect to $f$, after which we set $f=f_{i}{ }^{*}$ These derivatives are conveniently expressed as follows:

$$
\left.\frac{d}{d f} L_{i}^{(n)}(f)\right|_{f=f_{i}}=\sum_{j \neq i} 1 /\left(f_{i}-f_{j}\right)
$$

which is written more concisely, employing the notation

$$
1 /\left(f_{i}-f_{j}\right) \equiv \alpha_{i j}
$$

as

$$
L_{i}^{(n) \prime}\left(f_{i}\right)=\sum_{j \neq i} \alpha_{i j}
$$

Differentiating $L_{i}^{(n)}(f)$ twice and setting $f=f_{i}$, we obtain from (3),

$$
\left.\frac{d^{2} L_{i}^{(n)}(f)}{d f^{2}}\right|_{f=f_{i}}=2 \sum_{\substack{j \neq i \\ \text { and }}} \sum_{\substack{k \neq i \\ j \neq k}} 1 /\left(f_{i}-f_{j}\right)\left(f_{i}-f_{k}\right),
$$

the outside factor of 2 occurring because in every $(j, k)$ combination there will be a $(k, j)$ combination, $j \neq k$. The double summation occurring in $\left(6^{\prime \prime}\right)$ is avoided by employing the identity $2 \sum \sum \alpha_{i j} \alpha_{i k}=\left(\sum \alpha_{i j}\right)^{2}-\sum \alpha_{i j}{ }^{2}$ so that

$$
L_{i}^{(n) \prime \prime}\left(f_{i}\right)=\left(\sum_{j \neq i} \alpha_{i j}\right)^{2}-\sum_{j \neq i} \alpha_{i j}^{2}
$$

which from (4) is simply

$$
L_{i}^{(n) \prime \prime}\left(f_{i}\right)=\left\{L_{i}^{(n) \prime}\left(f_{i}\right)\right\}^{2}-\sum_{j \neq i} \alpha_{i j}^{2}
$$

For osculatory interpolation, following [7] p. 213, we define

$$
a_{i}=A_{i}^{2}
$$

which from (2") and (5) may be expressed as

$$
a_{i}=\left\{\prod_{j \neq i} \alpha_{i j}\right\}^{2}
$$

and

$$
b_{i}=-2 A_{i}^{2} L_{i}^{(n) \prime}\left(f_{i}\right),
$$

which from $\left(2^{\prime \prime}\right),(4)$ and (5) is expressible as

$$
b_{i}=-2\left\{\prod_{j \neq i} \alpha_{i j}\right\}^{2} \sum_{j \neq i} \alpha_{i j} .
$$

From (7) and (8) we define

$$
\alpha_{i}=a_{i} /\left(f-f_{i}\right)^{2}+b_{i} /\left(f-f_{i}\right),
$$

* For the occurrence of derivatives of Lagrangian coefficients in direct interpolation see [7] p. 213, for the osculatory case, and [3] p. 105, for the hyperosculatory case. 
and

$$
\beta_{i}=a_{i} /\left(f-f_{i}\right) .
$$

Finally for the $n$ points $x_{i}$ where we have both $f\left(x_{i}\right)$ and $f^{\prime}\left(x_{i}\right)$, we find for $x$ an approximation by the polynomial of the $(2 n-1)$-th degree in $f \equiv f(x)$, which is equal to $x_{i}$ at $f=f_{i}$ and whose derivative with respect to $f$ is equal to $x_{i}{ }^{\prime} \equiv$ $x^{\prime}\left(f_{i}\right)$ at $f=f_{i}$, according to

$$
x \sim \sum\left(\alpha_{i} x_{i}+\beta_{i} x_{i}{ }^{\prime}\right) / \sum \alpha_{i} .
$$

For hyperosculatory interpolation, following [3] p. 105, and taking into account $\left(2^{\prime \prime}\right),(4)$ and (5), we define

$$
\begin{aligned}
a_{i} & =\left\{\prod_{j \neq i} \alpha_{i j}\right\}^{3}, \\
b_{i} & =-3\left\{\prod_{j \neq i} \alpha_{i j}\right\}^{3} \sum_{j \neq i} \alpha_{i j},
\end{aligned}
$$

and a quantity $c_{i}$ which in standard notation is given by

$$
c_{i}=A_{i}^{3}\left[-\frac{3}{2} L_{i}^{(n) \prime \prime}\left(f_{i}\right)+6\left\{L_{i}^{(n) \prime}\left(f_{i}\right)\right\}^{2}\right],
$$

but when taking into account $\left(2^{\prime \prime}\right),(4),(5)$ and $(6)$, is expressible in present notation by

$$
c_{i}=\left\{\prod_{j \neq i} \alpha_{i j}\right\}^{3}\left[\frac{9}{2}\left\{\sum_{j \neq i} \alpha_{i j}\right\}^{2}+\frac{3}{2} \sum_{j \neq i} \alpha_{i j}^{2}\right] .
$$

Next, using (12)-(14), we define

$$
\begin{gathered}
\alpha_{i}=a_{i} /\left(f-f_{i}\right)^{3}+b_{i} /\left(f-f_{i}\right)^{2}+c_{i} /\left(f-f_{i}\right), \\
\beta_{i}=a_{i} /\left(f-f_{i}\right)^{2}+b_{i} /\left(f-f_{i}\right),
\end{gathered}
$$

and

$$
\gamma_{i}=a_{i} / 2\left(f-f_{i}\right) .
$$

Then for the $n$ points $x_{i}$ where we are given $f\left(x_{i}\right), f^{\prime}\left(x_{i}\right)$ and $f^{\prime \prime}\left(x_{i}\right)$, we find for $x$ an approximation by the polynomial of the $(3 n-1)$-th degree in $f$ which, together with its first and second derivative with respect to $f$, is equal to $x_{i}, x_{i}{ }^{\prime}$ and $x_{i}^{\prime \prime} \equiv-f^{\prime \prime}\left(x_{i}\right) /\left[f^{\prime}\left(x_{i}\right)\right]^{3}$ at $f=f_{i}$, according to

$$
x \sim \sum\left(\alpha_{i} x_{i}+\beta_{i} x_{i}{ }^{\prime}+\gamma_{i} x_{i}{ }^{\prime \prime}\right) / \sum \alpha_{i} .
$$

In using (11) or (18), when there are many inverse interpolations with all the values of $f$ being close to each other so that we have the same fixed points $f_{i}$, the quantities $a_{i}$ and $b_{i}$ in (7) and (8), or $a_{i}, b_{i}$ and $c_{i}$ in (12)-(14) have to be computed just once, to be used repeatedly in (9)-(11) or (15)-(18).

4. Application to Mathematical Table-Making. In a mathematical table where the inverse function will often be wanted, we might avoid the need for a separate table of the inverse function by supplementing the usual aids to direct interpolation (columns of differences or derivatives) with three, five, or even seven extra columns to facilitate osculatory or hyperosculatory inverse interpolation. For example, we might add three columns of just $x_{i}{ }^{\prime} \equiv 1 / f^{\prime}\left(x_{i}\right)$ with $a_{i}$ and $b_{i}$ defined by (7) 
and (8) to aid just osculatory inverse interpolation by (9)-(11), or five columns of $x_{i}{ }^{\prime}, x_{i}{ }^{\prime \prime} \equiv-f^{\prime \prime}\left(x_{i}\right) /\left[f^{\prime}\left(x_{i}\right)\right]^{3}, a_{i}, b_{i}$ and $c_{i}$ defined by (12)-(14) to help in just hyperosculatory inverse interpolation in (15)-(18), or even seven columns of $x_{i}{ }^{\prime}$, $x_{i}{ }^{\prime \prime}$, one set of functions $a_{i}, b_{i}$ defined by (7), (8) and another set of functions $a_{i}, b_{i}, c_{i}$, defined by (12)-(14), giving the user a choice of either osculatory or hyperosculatory inverse interpolation.

The use of these supplementary columns would not be restricted to tables of functions for just regularly spaced arguments $x_{i}$. Thus we may tabulate these auxiliary quantities $a_{i}, b_{i}$ and $c_{i}$ for tables having real arguments $x_{i}$ irregularly spaced or for tables having complex arguments in a Cartesian or polar grid. Even for osculatory or hyperosculatory direct interpolation in a table whose arguments are irregularly spaced points $x_{i}$, real or complex, if given $f_{i}^{\prime} \equiv f^{\prime}\left(x_{i}\right)$, or $f_{i}^{\prime}$ and $f_{i}^{\prime \prime} \equiv f^{\prime \prime}\left(x_{i}\right)$, we may tabulate $a_{i}, b_{i}$ by (7), (8), or $a_{i}, b_{i}, c_{i}$ by (12)-(14), and use $(9)-(11)$ or $(15)-(18)$, merely interchanging throughout in (5), (7)-(18) the variables $x_{i}$ with $f_{i}$ and $x$ with $f$.

In the functions $a_{i}, b_{i}$ and $c_{i}$, the choice of the number $n$ of fixed points should be, where feasible, sufficient to ensure full accuracy in the use of (11) or (18), which cannot be finer than the tabular uncertainty error of around $\epsilon / f^{\prime}\left(x_{i}\right)$, the $\epsilon$ being the error in the value of $f\left(x_{i}\right)$.

Convair Astronautics

San Diego, California

1. H. E. SALZER, "Formulas for inverse osculatory interpolation," NBS, Jn. of Res., v. 56, 1956, p. $51-54$.

2. H. E. SALZER, "Formulas for inverse osculatory interpolation in the complex plane," $N B S$, Jn. of Res., v. 59, 1957, p. 233-238.

3. H. E. SALzER, "Formulae for hyperosculatory interpolation, direct and inverse," Quart. Jn. of Mech. and Appl. Math., v. 12, 1959, p. 100-110.

4. H. E. SALzER, "A new formula for inverse interpolation," Bull., Amer. Math. Soc., v. 50,1944 , p. 513-516.

5. H. E. Salzer, "Inverse interpolation for eight-, nine-, ten-, and eleven-point direct interpolation," Jn. of Math. and Phys., v. 24, 1945, p. 106-108.

6. H. E. SALZER, "Formulas for direct and inverse interpolation of a complex function tabulated along equidistant circular arcs," Jn. of Math. and Phys., v. 24, 1945, p. 141-143.

7. H. E. SALZER, "New formulas for facilitating osculatory interpolation," NBS, Jn. of Res., v. 52, 1954, p. 211-216.

8. H. E. SALZER, "Osculatory interpolation in the complex plane," NBS, Jn. of Res., v. 54,1955 , p. 263-266. 